\title{
Asthma in Indiana - Using a Community Health Matrix to Determine Asthma Health Factors for Indiana Counties
}

\author{
Mikayla Burrell, Rachael Casey, MPH, Dennis Savaiano, PhD \\ Indiana University School of Medicine, Indiana Clinical and Translational Sciences Institute
}

Background and Hypothesis: Asthma and its appropriate treatment is a public health issue in Indiana that Indiana Joint Asthma Coalition (InJAC), a partnership within CTSI, is attempting to address. This is done through state-wide coalition building, which unifies efforts regarding asthma health and education and promotes interprofessional collaboration. Because time and resources are limited, InJAC must choose the areas that would benefit most from their focused work. A matrix was developed to establish the 10 counties with poorest asthma health and high vulnerability to social determinants to aid in this choice. We hypothesize that the 10 counties with the highest vulnerability to social determinants of health will have the worst asthma health.

Project Methods: Asthma health outcomes, contributing asthma-related variables, and social determinants of health were identified in all 92 counties in Indiana. Counties were compared by composite $z$ scores to determine the top 10 counties with the poorest health statistics for asthma and social determinants. In addition, qualitative data will be used to identify local health coalitions that have the capacity and desire to work with InJAC to improve asthma treatment. InJAC will begin sessions with these counties to determine if long-term, sustainable, health promotions are feasible.

Results: The top 10 counites that were identified as having the poorest asthma health and factors were Lake, Grant, Madison, Marion, Huntington, Vanderburgh, Howard, La Porte, Blackford, and Noble. The top 10 counties with highest vulnerability to social determinants were Owen, Ripley, Daviess, La Grange, Fayette, Wayne, Elkhart, Newton, Switzerland, and Marion.

Potential Impact: The data from this matrix will help direct InJAC to the areas of Indiana with the most need for asthma coalition efforts. This will be done through improvement on education, awareness, and quality of care based on the Indiana State Asthma Plan. 